\title{
Dynamic thermoelectric and heat transport in mesoscopic capacitors
}

\author{
Jong Soo Lim, ${ }^{1,2}$ Rosa López, ${ }^{1,3}$ and David Sánchez ${ }^{1,3}$ \\ ${ }^{1}$ Instituto de Física Interdisciplinar y Sistemas Complejos IFISC (UIB-CSIC), E-07122 Palma de Mallorca, Spain \\ ${ }^{2}$ School of Physics, Korea Institute for Advanced Study, Seoul 130-722, Korea \\ ${ }^{3}$ Departament de Física, Universitat de les Illes Balears, E-07122 Palma de Mallorca, Spain
}

(Received 30 July 2013; published 18 November 2013)

\begin{abstract}
We discuss the low-frequency response of charge and heat transport to oscillatory voltage and temperature shifts in mesoscopic capacitors. We obtain, within scattering theory, generic expressions for the quantum admittances up to second order in the ac frequencies in terms of electric, thermoelectric, and heat capacitances and relaxation resistances. Remarkably, we find that the thermocurrent can lead or lag the applied temperature depending on the gate voltage applied to a quantum $R C$ circuit. Furthermore, the relaxation resistance for cross terms becomes nonuniversal as opposed to the purely electric or thermal cases.
\end{abstract}

DOI: 10.1103/PhysRevB.88.201304

PACS number(s): 73.23.-b, 72.15.Jf, 62.25.De, 65.80.-g

Introduction. Time-dependent charge transport in quantum conductors subject to ac electric fields provides insight into electronic dynamics at nanoscale dimensions. ${ }^{1}$ For low frequencies, the dynamics of a mesoscopic conductor is characterized by the $R_{G} C_{G}$ time, where $C_{G}$ is the quantum capacitance and $R_{G}$ is the charge relaxation resistance. ${ }^{2}$ Whereas the former generally depends on energy, the latter unexpectedly attains the constant value of $h / 2 e^{2}$ in a singlechannel quantum capacitor. ${ }^{1}$ These predictions are confirmed by ac measurements in mesoscopic $R C$ circuits. $^{3}$ Further developments have led to the experimental demonstration of coherent single-electron emitters. ${ }^{4}$ This achievement has spurred an enormous interest in both the fundamentals of time-resolved electronic transport (both experimentally ${ }^{5-7}$ and theoretically ${ }^{8-15}$ ) and its applications to, e.g., metrology ${ }^{16}$ and quantum information processing, ${ }^{17}$ just to mention a few.

Electronic current, however, can also be driven by thermal gradients. In the stationary case, the Seebeck effect leads to the generation of thermovoltages in response to applied temperature differences in open circuits. ${ }^{18}$ While dc thermopower has been extensively investigated in nanostructures, ${ }^{19-23}$ the ac Seebeck effect has received little attention to date. The subject is interesting for several reasons. First, treating voltage and thermal driving fields on an equal footing opens up the door to not only electrical but also thermodynamic characterizations of mesoscopic systems. In fact, ac calorimetry techniques have been successfully applied to superconducting loops. ${ }^{24}$ What can be learned from an analogous experiment with normal conductors? Second, quantum refrigeration devices based on the Peltier effect (reciprocal to Seebeck) typically use static currents. ${ }^{25}$ What can we expect in the ac regime of transport? Third, how does Coulomb interaction renormalize the $R C$ parameters in a thermoelectric device? These are the kind of questions we want to address in this work.

Consider a multichannel mesoscopic conductor coupled to a single terminal as in Fig. 1(a). The sample is driven out of equilibrium with oscillating voltages ${ }^{3} \delta V(\omega)$ and temperatures ${ }^{26} \delta T(\omega)$ applied to the reservoir. The driving fields operate with regard to an equilibrium state described by the chemical potential $\mu$ and the base temperature $T_{0}$. Hence, the linear-response electric $\delta I$ and heat $\delta J$ currents are given (a)
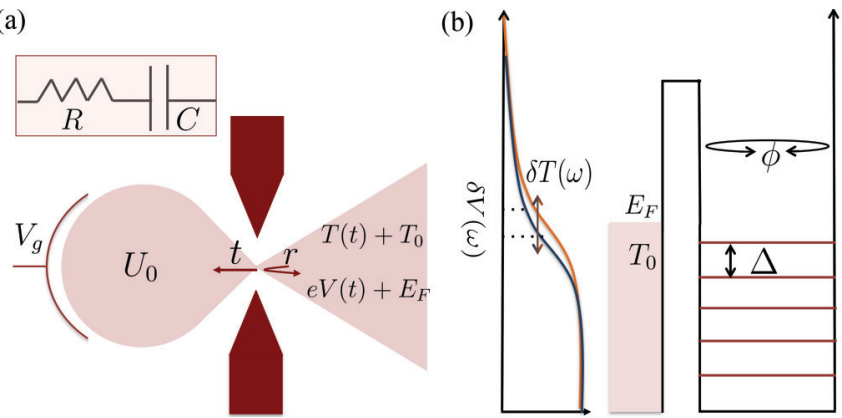

FIG. 1. (Color online) (a) Sketch of a mesoscopic capacitor: a large dot attached to a reservoir with time-dependent (oscillatory) temperature $T(t)$ and voltage $V(t)$ with respect to the base temperature $T_{0}$ and chemical potential $\mu \simeq E_{F}$. The coupling region is a single-mode quantum point contact with transmission $t$ and reflection $r$ amplitudes modulated with external gates (dark shaded areas). Interactions are modeled with a homogeneous potential $\delta U_{0}$ that reacts to a change in a nearby gate contact coupled via a geometrical capacitance $C$. Electric, thermoelectric, and heat properties of the capacitor are described at low-frequency $\omega$ with an equivalent $R C$ circuit, where $R_{\mathcal{A}}$ and $C_{\mathcal{A}}$ depend on the transport type (charge, energy, or both). (b) Energy diagram of the mesoscopic capacitor. The Fermi distribution function in the reservoir changes with the oscillatory voltages. The dot contains many levels with mean spacing $\Delta . \phi$ is the electronic accumulated phase in a single turn around the dot.

by

$$
\left(\begin{array}{l}
\delta I \\
\delta J
\end{array}\right)=\left(\begin{array}{ll}
G(\omega) & L(\omega) \\
M(\omega) & K(\omega)
\end{array}\right)\left(\begin{array}{l}
\delta V \\
\delta T
\end{array}\right)
$$

where the $2 \times 2$ Onsager matrix includes diagonal elements [electric $G(\omega)$ and thermal $K(\omega)$ admittances] and nondiagonal coefficients [thermoelectric $L(\omega)$ and electrothermal $M(\omega)$ admittances]. The latter are related by reciprocity. ${ }^{27}$

In a low-frequency expansion, $G(\omega)=-i \omega C_{G}+$ $\omega^{2} C_{G}^{2} R_{G}$, the imaginary part of the electric admittance provides information on the electric capacitance or emittance of the system, whereas its real part is directly related to the dissipation in the conductor. When transport is phase coherent, $R_{G}=h / 2 e^{2}$ takes a universal value independently of the transmission value and it scales with $N$ the number 
of propagating channels. ${ }^{1}$ Below, we demonstrate that the low-temperature thermal relaxation resistance also becomes quantized but, surprisingly, its thermoelectric analog is always nonuniversal. Importantly, our gauge-invariant theory also includes the effect of Coulomb interactions taking into account the charges that pile up when the sample is driven out of equilibrium by oscillatory voltage and thermal biases. We will illustrate our ac thermoelectrical scattering theory with an application to a quantum capacitor system.

Scattering formalism. We present a scattering approach for coupled charge and energy transport. In general, the transport properties of a multichannel mesoscopic capacitor are described by the scattering matrix $S$ with elements $s_{n m}[E, U(\vec{r})]$ that relate the outgoing current amplitude in channel $n$ to the incoming current amplitude in channel $m$ for a carrier with a given energy $E$. The matrix is also a function of the internal potential landscape $U(\vec{r})$ built up inside the conductor. Quite generally, $U(\vec{r})$ is a function of the applied electrical and thermal biases. ${ }^{28,29}$ Let us for the moment focus on the noninteracting case. Then, the time-dependent charge and heat current operators are given by $\hat{I}=(e / h) \int d E d E^{\prime} \exp \left[i\left(E-E^{\prime}\right) t / \hbar\right]\left[a^{\dagger}(E) a\left(E^{\prime}\right)-\right.$ $\left.b^{\dagger}(E) b\left(E^{\prime}\right)\right] \quad$ and $\quad \hat{J}=(1 / 2 h) \int d E d E^{\prime}\left(E+E^{\prime}-\right.$ $2 \mu) \exp \left[i\left(E-E^{\prime}\right) t / \hbar\right]\left[a^{\dagger}(E) a\left(E^{\prime}\right)-b^{\dagger}(E) b\left(E^{\prime}\right)\right]$, where $a=\left(a_{1}, a_{2}, \ldots, a_{N}\right)^{\dagger}$ and $b=\left(b_{1}, b_{2}, \ldots, b_{N}\right)^{\dagger}$ denote incoming and outgoing annihilation operators in the lead with $N$ channels and fulfill $b_{n}(E)=\sum_{m} s_{n m}(E) a_{m}(E)$. We hereafter take $\mu \simeq E_{F}$ (the Fermi energy) independently of $T_{0}$, which is a good approximation at low temperature, the regime of our interest.

The linear response of the electrical and heat currents to the ac external perturbations in Eq. (1) are expressed as $G(\omega)=$ $\delta I(\omega) / \delta V(\omega), L(\omega)=\delta I(\omega) / \delta T(\omega), M(\omega)=\delta J(\omega) / \delta V(\omega)$, and $K(\omega)=\delta J(\omega) / \delta T(\omega)$, where $I=\langle\hat{I}\rangle$ and $J=\langle\hat{J}\rangle$. The admittances can be obtained from the Kubo formulas, ${ }^{30-32}$

$$
\begin{aligned}
G(\omega) & =\frac{1}{\hbar \omega} \int_{0}^{\infty} d t e^{i\left(\omega+i 0^{+}\right) t}\langle[\hat{I}(t), \hat{I}(0)]\rangle, \\
K(\omega) & =\frac{1}{\hbar \omega T_{0}} \int_{0}^{\infty} d t e^{i\left(\omega+i 0^{+}\right) t}\langle[\hat{J}(t), \hat{J}(0)]\rangle, \\
L(\omega) & =\frac{1}{\hbar \omega T_{0}} \int_{0}^{\infty} d t e^{i\left(\omega+i 0^{+}\right) t}\langle[\hat{I}(t), \hat{J}(0)]\rangle, \\
M(\omega) & =\frac{1}{\hbar \omega} \int_{0}^{\infty} d t e^{i\left(\omega+i 0^{+}\right) t}\langle[\hat{J}(t), \hat{I}(0)]\rangle .
\end{aligned}
$$

By inserting the above expressions for $\hat{I}$ and $\hat{J}$ in Eq. (2d), we find the reciprocity relation $M(\omega)=T_{0} L(\omega)$, as expected. In the presence of an external magnetic field $B$, reciprocity becomes $M(\omega, B)=T_{0} L(\omega,-B)$. An important remark is now in order. Concerns have been recently raised about the validity of the fluctuation-dissipation theorem applied to heat transport. ${ }^{33}$ In fact, Refs. 33 and 34 find a nonvanishing term for the equilibrium heat-heat correlation function at $T_{0} \rightarrow 0$, which is incompatible with the expected behavior of $K(\omega)$. However, this term is associated to scattering events that connect two different terminals and can therefore be safely ignored in our single-lead quantum capacitor system.
Inserting the charge and current operator expressions in Eq. (2), we find

$$
\begin{aligned}
G(\omega) & =\frac{e^{2}}{h} \int d E \operatorname{Tr} A(E, E+\hbar \omega) \bar{F}(E, \omega), \\
K(\omega) & =\frac{1}{h T_{0}} \int d E E_{\omega}^{2} \operatorname{Tr} A(E, E+\hbar \omega) \bar{F}(E, \omega), \\
L(\omega) & =\frac{e}{h T_{0}} \int d E E_{\omega} \operatorname{Tr} A(E, E+\hbar \omega) \bar{F}(E, \omega),
\end{aligned}
$$

where the trace is over the transverse channels, $E_{\omega}=\left(E+\hbar \omega / 2-E_{F}\right), A(E, E+\hbar \omega)=1-S^{\dagger}(E) S(E+$ $\hbar \omega)$, and $\bar{F}(E, \omega)=\left[f_{0}(E)-f_{0}(E+\hbar \omega)\right] / \hbar \omega$ with $f_{0}=$ $1 /\left\{1+\exp \left[\left(E-E_{F}\right) / k_{B} T_{0}\right]\right\}$ the Fermi function of the equilibrium reference state.

Low-frequency analysis. The quantum capacitor exhibits pure ac response. Then, we expand Eq. (3) in powers of $\omega$. The leading-order contributions have the following functional form for $\mathcal{A}=G, K$, and $L$ :

$$
\mathcal{A}=-i \omega C_{\mathcal{A}}+\omega^{2} C_{\mathcal{A}}^{2} R_{\mathcal{A}}+O\left(\omega^{3}\right),
$$

where

$$
C_{\mathcal{A}}=g_{\mathcal{A}} \int d E\left(-\frac{\partial f_{0}}{\partial E}\right)\left(E-E_{F}\right)^{\lambda} \rho(E)
$$

and

$$
R_{\mathcal{A}}=\frac{r_{\mathcal{A}} \int d E\left(E-E_{F}\right)^{\lambda}\left(-\frac{\partial f_{0}}{\partial E}\right) \operatorname{Tr}\left[\left(S^{\dagger} \partial_{E} S\right)^{2}\right]}{\left\{\int d E\left(E-E_{F}\right)^{\lambda}\left(-\frac{\partial f_{0}}{\partial E}\right) \operatorname{Tr}\left[S^{\dagger} \partial_{E} S\right]\right\}^{2}},
$$

where $g_{\mathcal{A}}$ and $r_{\mathcal{A}}$ are constants to be specified below.

In Eq. (5), we define $\rho(E)=\operatorname{Tr}\left[S^{\dagger} \partial_{E} S\right] / 2 \pi i$ as the density of states for the capacitor plate. ${ }^{8}$ Notice that our formalism considers scattering states only. Thus, contributions to $\rho(E)$ due to bound states are not included. Furthermore, the index $\lambda$ in Eqs. (5) and (6) denotes the type of transport: $\lambda=0$ (electric), $\lambda=1$ (thermoelectric), or $\lambda=2$ (thermal). We now discuss their main properties.

Electric admittance. For $\mathcal{A}=G$, we have $g_{G}=e^{2}$. In this case, $C_{G}$ is a quantum capacitance given by the density of states and can be accessed experimentally. ${ }^{35}$ Equations (5) and (6) are generally valid for an arbitrary $N$-channel conductor. However, we now focus on the $N=1$ case because it is the experimentally relevant situation. We model the mesoscopic capacitor as a quantum dot coupled to a reservoir ${ }^{3,4}$ by means of a single-mode quantum point contact with energy-dependent transmission probability $\mathcal{T}(E)=1 /\{1+$ $\left.\exp \left[-\left(E-\varepsilon_{0}\right) / \Omega\right]\right\}$, where $\Omega$ is a characteristic energy scale of the potential curvature and $\varepsilon_{0}$ is the transverse energy of the first propagating mode in the point contact. A strong magnetic field is applied perpendicular to the dot and transport occurs along edge states. Let $\phi=2 \pi E / \Delta$ be the phase that an electron picks up after a single turn around the dot, see Fig. 1(b). Then, the scattering amplitude is ${ }^{3,4} S(E)=$ $[\sqrt{1-\mathcal{T}}-\exp (i \phi)] /[1-\sqrt{1-\mathcal{T}} \exp (i \phi)]$, from which the density of states $\rho(E)$ easily follows. In Fig. 2(a), we plot $C_{G}$ as a function of the energy difference $E_{F}-\varepsilon_{0}$. Each peak is associated with a level in the quantum dot. Finally, the charge relaxation resistance $R_{G}$ is obtained from Eq. (6) by setting $\lambda=0$ and $r_{G}=h / 2 e^{2}$. In the single-channel case, one 

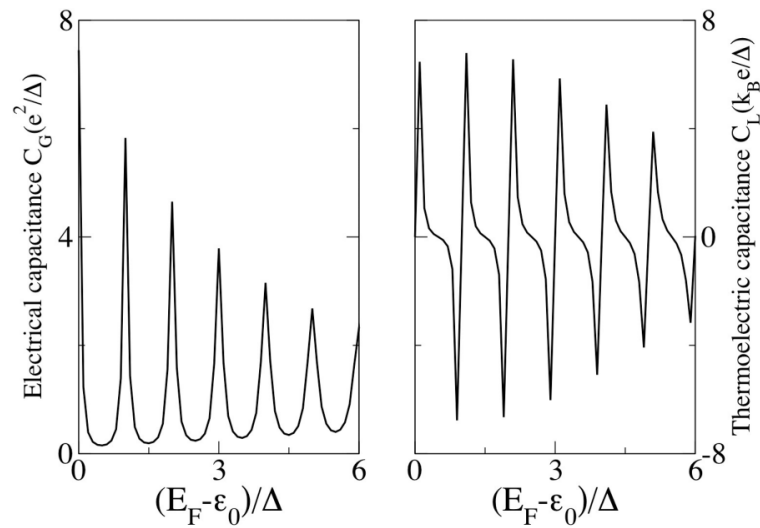

FIG. 2. (a) Charge and (b) thermoelectric capacitances as a function of the gate voltage for the quantum system depicted in Fig. 1. Parameters: $\varepsilon_{0}=\Delta, \Omega=3 \Delta, T_{0}=\Delta / 10$.

recovers the universal value $R_{G}=r_{G}$ valid at sufficiently low temperatures.

Heat admittance. $K(\omega)=-i \omega C_{K}+\omega^{2} C_{K}^{2} R_{K}$ is found from Eqs. (5) and (6) with $g_{K}=1 / T_{0}$ and $r_{K}=h T_{0} / 2$, respectively. Similarly to the electric case, the imaginary part is related to a capacitance. Here, $C_{K}$ has the dimensions of a heat capacity. In fact, $C_{K}$ is the measured quantity in a low-frequency ac calorimetry experiment. ${ }^{24}$ At low temperature, we find, to leading order in a Sommerfeld expansion, $C_{K}=\pi^{2} k_{B}^{2} T_{0} \rho\left(E_{F}\right) / 3$. Higher orders can be safely neglected for temperatures smaller than the energy variation of $\rho$, which can be estimated as the mean level spacing $\Delta$. Therefore, the density of states determines not only the charge (quantum) capacitance, but also its energy counterpart. More interestingly, the dissipative part of $K(\omega)$ becomes, at low temperature,

$$
R_{K}=\frac{3 h}{2 \pi^{2} k_{B}^{2} T_{0}} .
$$

This result has a simple interpretation. We recall that $G_{t h}=$ $\pi^{2} k_{B}^{2} T_{0} / 3 h$ is the thermal conductance quantum, recently measured in a suspended nanostructure. ${ }^{36}$ Hence, $R_{K}=1 /\left(2 G_{t h}\right)$ is the associated thermal relaxation resistance for a singlemode mesoscopic capacitor. Equation (7) is also universal at low temperature. We note in passing that the characteristic time $\tau_{K}=R_{K} C_{K}=h \rho\left(E_{F}\right) / 2$ is always positive, as it should be, and equals the $R C$ time for pure electric transport, $\tau_{G}=$ $R_{G} C_{G}=h \rho\left(E_{F}\right) / 2 .{ }^{1}$ Furthermore, Eq. (7) scales as $1 / N$ with the number of conducting channels, as occurs for the electrical case, because the numerator of Eq. (6) goes as $N$ and its denominator as $N^{2}$.

Thermoelectric admittance. Coupled charge and energy transport is governed at low frequencies by $L(\omega)=-i \omega C_{L}+$ $\omega^{2} C_{L}^{2} R_{L}$ with $g_{L}=e / T_{0}$ and $r_{L}=h T_{0} / 2 e$ in Eqs. (5) and (6) for $\lambda=1$. At low temperature, the thermoelectric capacitance becomes

$$
C_{L}=\frac{\pi^{2}}{3} e k_{B}^{2} T_{0} \rho^{\prime}\left(E_{F}\right)
$$

Remarkably, $C_{L}$ can take positive or negative values for a conductor with a density of states that strongly depends on energy (e.g., a quantum dot with quasilocalized levels). This

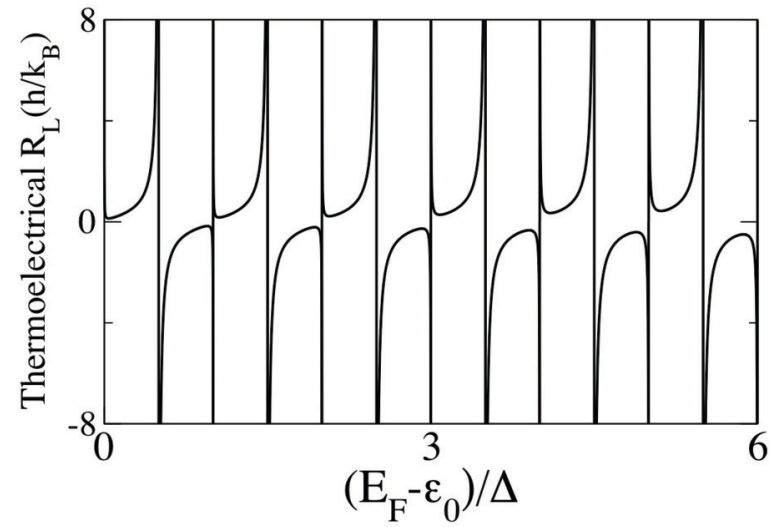

FIG. 3. Thermoelectrical relaxation resistance as a function of the gate voltage. We take the same parameters as in Fig. 2.

is in sharp contrast with the charge capacitance, which takes positive values only, as can be seen from its low- $T_{0}$ expansion: $C_{G}=e^{2} \rho\left(E_{F}\right)$. As a consequence, while electric currents lead $\delta V(\omega)$ by $\pi / 2$ in a capacitor, the thermocurrents lead or lag $\delta T$ depending on the sign of $C_{L}$. This observation is confirmed for our dot capacitor, in which we observe periodic changes of sign whenever $E_{F}-\varepsilon_{0}$ crosses an energy level in the dot; see Fig. 2(b).

In the Seebeck effect, a voltage is generated in response to a temperature shift under the condition $I=0$. Thus, the Seebeck coefficient is at linear response $S=-L / G .{ }^{18} \mathrm{We}$ find in the low-frequency limit that $S$ is given by a ratio of capacitances: $S(\omega)=-C_{L} / C_{G}+O(\omega)$. From our results in Fig. 2, we expect a strong dependence of $S$ as a function of energy in a mesoscopic capacitor. At low temperature, we find the Mott formula ${ }^{37} S=\left.\left(\pi^{2} k_{B}^{2} T_{0} / 3 e\right) \partial_{E} \ln \rho(E)\right|_{E=E_{F}}$. Note that the same energy dependence is obtained from a study of the Peltier effect, since the Peltier coefficient is simply $\Pi=T_{0} S$ by reciprocity. ${ }^{19}$

We find, for the thermoelectric relaxation resistance $R_{L}$ at low temperature,

$$
R_{L}=\left.\frac{3 h}{e \pi^{2} k_{B}^{2} T_{0}} \frac{\rho(E)}{\rho^{\prime}(E)}\right|_{E=E_{F}},
$$

which again scales as $1 / N$ for a $N$-channel conductor. Unlike the charge relaxation resistance and $R_{K}$ in the limit $T_{0} \rightarrow 0$, the resistance $R_{L}$ is always a nonuniversal function that depends on the sample details. Surprisingly enough, we can recast the inverse of Eq. (9) in a more familiar form since $G_{L} \equiv R_{L}^{-1}=\left.e G_{t h} \partial_{E} \ln \rho(E)\right|_{E=E_{F}}$ resembles the Mott formula for the dc thermopower ${ }^{37}$ but applied to a seemingly unrelated quantity. In Fig. 3, we plot $R_{L}$ for the mesoscopic capacitor as a function of the gate voltage. As expected, the thermoelectric relaxation resistance never reaches a constant value. Note that the divergent behavior of $R_{L}$ in Fig. 3 occurs whenever the $\rho^{\prime}\left(E_{F}\right)$ vanishes. Obviously the measurable quantity $\tau_{L}=R_{L} C_{L}=h \rho\left(E_{F}\right)$ is always finite and nonzero. Interestingly, the time scale for cross charge-energy transport is two times larger than purely charge or heat characteristic times. Then, for frequencies around $\tau_{G}^{-1}, \tau_{L}^{-1}$, or $\tau_{K}^{-1}$ (all of the same order), the $\omega^{2}$ term becomes comparable to the linear-in- $\omega$ term, allowing us to probe the characteristic times. 
For a quantum capacitor, we approximate $\rho\left(E_{F}\right) \sim 1 / \Delta$ and estimate $\omega \sim 1 \mathrm{GHz}$ for a typical value $\Delta=10 \mu \mathrm{eV}$. This frequency is not far from the scope of present technology for both voltage ${ }^{3}$ and temperature ${ }^{38}$ biases.

Interactions. A real calculation of admittance responses requires knowledge of the charge distribution inside the sample when the conductor is driven out of equilibrium by ac signals. ${ }^{2}$ Thus, we need to discuss screening effects. As a first approximation, we treat interactions within a mean-field theory.

Let $\delta U_{0}$ be the internal potential in the conductor away from its equilibrium value. For definiteness, we assume that $\delta U_{0}$ is spatially homogeneous [see Fig. 1(a) for an example]. The extension to inhomogeneous fields is straightforward. ${ }^{28,29}$ In the presence of interactions, the linear-response current becomes

$$
\delta I=G(\omega) \delta V+L(\omega) \delta T+\Pi(\omega) \delta U_{0},
$$

with $\Pi(\omega)$ the screening function. Because $\delta I$ is invariant under a global voltage shift, $\Pi(\omega)=-G(\omega)$. On the other hand, the conductor is coupled to nearby gates via capacitance couplings. In the homogeneous case, we consider a single electric capacitance $C$. Then, the ac current is $\delta I=-i \omega C \delta U_{0}$ and, using current conservation in Eq. (10), we find

$$
\delta U_{0}=\frac{G(\omega) \delta V+L(\omega) \delta T}{G(\omega)-i \omega C} .
$$

We emphasize that the internal potential changes in response not only to voltage variations but also to temperature shifts. ${ }^{29}$

Substituting Eq. (11) into Eq. (10), we obtain the electric and thermoelectric impedances, $\mathcal{G}^{-1}(\omega)=G^{-1}(\omega)+$ $i / \omega C$ and $\mathcal{L}^{-1}(\omega)=L^{-1}(\omega)+i G(\omega) / \omega C L(\omega)$, respectively. Similarly, the interacting ac responses for the heat flow to oscillatory temperatures and voltages are $\mathcal{K}(\omega)=$ $K(\omega)-L(\omega) M(\omega) /[G(\omega)-i \omega C]$ and $\mathcal{M}(\omega)=M(\omega)-$ $G(\omega) M(\omega) /[G(\omega)-i \omega C]$, with the latter obeying reciprocity $\left(\mathcal{M}=T_{0} \mathcal{L}\right)$. Importantly, the four admittances constitute a gauge-invariant theory.

Previous expressions can be further simplified in the low-frequency limit. We find that the thermoelectrical response $\mathcal{L}(\omega)=\left(-i \omega C_{L}^{\mu}+\omega^{2} C_{L} C_{L}^{\mu} R_{L}\right)$ is expressed in terms of a renormalized thermoelectric capacitance $C_{L}^{\mu}=C_{L} C_{G}^{\mu} / C_{G}$, where the electrochemical capacitance $C_{G}^{\mu}$ is given by the geometrical and quantum capacitances in series: $\left(C_{G}^{\mu}\right)^{-1}=C^{-1}+C_{G}^{-1}$. Finally, the interacting heat admittance reads $\mathcal{K}(\omega)=$ $-i \omega C_{K}^{\mu}+\omega^{2}\left[C_{K}^{2} R_{K}-\left(C_{L} R_{L}+C_{M} R_{M}\right) C_{L} C_{M} /\left(C+C_{G}\right)\right]$, where the renormalized heat capacitance is $C_{K}^{\mu}=C_{K}-C_{M} C_{L} / C$.

Conclusion. We have presented a scattering theory for the dynamical response of heat and electrical currents to oscillatory electrical and thermal signals applied to a mesoscopic capacitor. Our model includes interactions and focuses on the low-frequency properties of charge and energy transport. Importantly, we have found that the offdiagonal admittance matrix elements show crucial differences with the pure electric or thermal conductances: positive or negative phase delays and sample-dependent relaxation resistances. Our results are relevant both for thermodynamic characterizations of nanosystems and for prospect applications of thermoelectric nanodevices operating in the time domain.

Acknowledgments. We thank M. Büttiker and J. Splettstoesser for useful comments. This work was supported by MINECO Grants No. FIS2011-23526 and No. CSD200700042 (CPAN).
${ }^{1}$ M. Büttiker, A. Prêtre, and H. Thomas, Phys. Lett. A 180, 364 (1993).

${ }^{2}$ M. Büttiker, A. Prêtre, and H. Thomas, Phys. Rev. Lett. 70, 4114 (1993).

${ }^{3}$ J. Gabelli, G. Fève, J.-M. Berroir, B. Plaçais, A. Cavanna, B. Etienne, Y. Jin, and D. C. Glattli, Science 313, 499 (2006).

${ }^{4}$ G. Fève, A. Mahé A, J. M. Berroirm, T. Kontos, B. Plaçais, D. C. Glattli, A. Cavanna, B. Etienne, and Y. Jin, Science 316, 1169 (2007).

${ }^{5}$ S. J. Chorley, J. Wabnig, Z. V. Penfold-Fitch, K. D. Petersson, J. Frake, C. G. Smith, and M. R. Buitelaar, Phys. Rev. Lett. 108, 036802 (2012).

${ }^{6}$ J. Basset, H. Bouchiat, and R. Deblock, Phys. Rev. B 85, 085435 (2012).

${ }^{7}$ T. Frey, P. J. Leek, M. Beck, J. Faist, A. Wallraff, K. Ensslin, T. Ihn, and M. Büttiker, Phys. Rev. B 86, 115303 (2012).

${ }^{8}$ S. E. Nigg, R. López, and M. Büttiker, Phys. Rev. Lett. 97, 206804 (2006).

${ }^{9}$ J. Wang, B. Wang, and H. Guo, Phys. Rev. B 75, 155336 (2007).

${ }^{10}$ Z. Ringel, Y. Imry, and O. Entin-Wohlman, Phys. Rev. B 78, 165304 (2008).
${ }^{11}$ M. Moskalets, P. Samuelsson, and M. Büttiker, Phys. Rev. Lett. 100, 086601 (2008).

${ }^{12}$ C. Mora and K. Le Hur, Nat. Phys. 6, 697 (2010).

${ }^{13}$ A. Cottet, C. Mora, and T. Kontos, Phys. Rev. B 83, 121311(R) (2011).

${ }^{14}$ M. Lee, R. López, M.-S. Choi, T. Jonckheere, and T. Martin, Phys. Rev. B 83, 201304(R) (2011).

${ }^{15}$ F. Battista, M. Moskalets, M. Albert, and P. Samuelsson, Phys. Rev. Lett. 110, 126602 (2013).

${ }^{16}$ J. P. Pekola, O.-P. Saira, V. F. Maisi, A. Kemppinen, M. Möttönen, Yu. A. Pashkin, and D. V. Averin, Rev. Mod. Phys. 85, 1421 (2013).

${ }^{17}$ J. Splettstoesser, M. Moskalets, and M. Büttiker, Phys. Rev. Lett. 103, 076804 (2009).

${ }^{18}$ Thermoelectrics Handbook. Macro to Nano, edited by D. M. Rowe (CRC, Boca Raton, FL, 2006).

${ }^{19}$ L. W. Molenkamp, Th. Gravier, H. van Houten, O. J. A. Buijk, M. A. A. Mabesoone, and C. T. Foxon, Phys. Rev. Lett. 68, 3765 (1992).

${ }^{20}$ A. S. Dzurak, C. G. Smith, C. H. W. Barnes, M. Pepper, L. MartínMoreno, C. T. Liang, D. A. Ritchie, and G. A. C. Jones, Phys. Rev. B 55, R10197 (1997). 
${ }^{21}$ P. Reddy, S.-Y. Jang, R. A. Segalman, and A. Majumdar, Science 315, 1568 (2007).

${ }^{22}$ R. Scheibner, M. König, D. Reuter, A. D. Wieck, C. Gould, H. Buhman, and L. W. Molenkamp, New J. Phys. 10, 083016 (2008).

${ }^{23}$ J. Matthews, D. Sánchez, M. Larsson, and H. Linke, Phys. Rev. B 85, 205309 (2012).

${ }^{24}$ O. Bourgeois, S. E. Skipetrov, F. Ong, and J. Chaussy, Phys. Rev. Lett. 94, 057007 (2005).

${ }^{25}$ F. Giazotto, T. T. Heikkilä, A. Luukanen, A. M. Savin, and J. P. Pekola, Rev. Mod. Phys. 78, 217 (2006).

${ }^{26}$ F. Chen, J. C. Cooley, W. L. Hults, and J. L. Smith, Rev. Sci. Instrum. 72, 4201 (2001).

${ }^{27}$ L. Onsager, Phys. Rev. 37, 405 (1931).

${ }^{28}$ M. Büttiker, J. Phys.: Condens. Matter 5, 9361 (1993).
${ }^{29}$ D. Sánchez and R. López, Phys. Rev. Lett. 110, 026804 (2013).

${ }^{30}$ G. D. Mahan, Many-Particle Physics (Plenum, New York, 1990), Sec. 3.9.

${ }^{31}$ H. B. Callen and T. A. Welton, Phys. Rev. 83, 34 (1951).

${ }^{32}$ R. Kubo, J. Phys. Soc. Jpn. 12, 570 (1957).

${ }^{33}$ D. V. Averin and J. P. Pekola, Phys. Rev. Lett. 104, 220601 (2010).

${ }^{34}$ D. Sergi, Phys. Rev. B 83, 033401 (2011).

${ }^{35}$ T. P. Smith, W. I. Wang, and P. J. Stiles, Phys. Rev. B 34, 2995 (1986).

${ }^{36}$ K. Schwab, E. A. Henriksen, J. M. Worlock, and M. L. Roukes, Nature (London) 404, 974 (2000).

${ }^{37}$ M. Cutler and N. F. Mott, Phys. Rev. 181, 1336 (1969).

${ }^{38}$ E. Shoifet, Y. Z. Chua, H. Huth, and C. Schick, Rev. Sci. Instrum 84, 073903 (2013). 